\title{
Imaging in Chronic Pancreatitis
}

\author{
Shikha Sood ${ }^{1}$ Devasenathipathy Kandasamy² Raju Sharma²
}

\author{
${ }^{1}$ Department of Radiodiagnosis, Indira Gandhi Medical College, \\ Shimla, India \\ 2Department of Radiodiagnosis, All India Institute of Medica \\ Sciences, New Delhi, India
}

J Gastrointestinal Abdominal Radiol ISGAR 2020;3:21-27

\begin{abstract}
Address for correspondence Devasenathipathy Kandasamy, MD, DNB, FRCR, Department of Radiodiagnosis, All India Institute of Medical Sciences, New Delhi 110029, India (e-mail: devammc@gmail.com).
\end{abstract}

\begin{abstract}
Keywords

- chronic pancreatitis

$-\mathrm{CT}$

$-M R I$

Chronic pancreatitis (CP) is a chronic progressive process which can cause irreversible damage to both the exocrine and endocrine systems of pancreas. There are various imaging modalities available to evaluate CP of which ultrasonography (USG), computed tomography (CT), and magnetic resonance imaging (MRI) are most commonly used. USG is used as a screening modality which can show various changes of CP and its complications. However, the information obtained from USG can be limited because of the patient's body habitus. CT is the workhorse modality which can provide comprehensive information and it is the best modality to detect calcification. MRI, on the other hand, is excellent in demonstrating the pancreatic parenchyma and the ductal system. Alcohol-related pancreatitis is the commonest cause which can show gland atrophy, ductal dilatation, and calcifications. Other less common causes include hereditary and genetic mutation-related pancreatitis. There are other special types of pancreatitis such as autoimmune pancreatitis and groove pancreatitis that have characteristic features on imaging.
\end{abstract}

\section{Introduction}

Chronic pancreatitis (CP) occurs because of chronic progressive, inflammatory, and fibrotic changes in the gland which lead to irreversible morphological changes as well as exocrine and endocrine insufficiency. ${ }^{1}$ Clinically, patients of $\mathrm{CP}$ present with abdominal pain, malabsorption, malnutrition, and diabetes. Alcoholism is a leading cause, while other risk factors include genetic, smoking, hypercalcemia, high protein diet, periampullary neoplasms, and abnormal pancreaticobiliary junction. ${ }^{2}$ Other rare causes include autoimmune pancreatitis, hereditary pancreatitis, and chronic renal failure (CRF). Gallstones, the major cause of acute pancreatitis, are not a risk factor for chronic pancreatitis.

Several imaging modalities like abdominal radiograph, ultrasonography (USG), computed tomography (CT), magnetic resonance imaging (MRI), and Endoscopic retrograde cholangiopancreatography (ERCP) are used to study the morphology and to some extent the functioning of the pancreas. Newer techniques like pancreatic CT perfusion, secretin stimulated magnetic resonance cholangiopancreatography (S-MRCP), endoscopic ultrasound (EUS), and shear wave elastography (SWE) help in early diagnosis and management. Besides imaging, fecal elastase is one of the common indirect methods used to evaluate the exocrine function of the pancreas. ${ }^{3}$ There is a direct and more invasive method known as tubed secretin test which necessitates prolonged duodenal intubation and the stimulated pancreatic juice is serially collected over a period of time. ${ }^{4,5}$ This test is tedious, time consuming, and available only in few centers. S-MRCP, on the other hand, can be used as one-stop shop modality to evaluate both morphology, as well as the exocrine function, of pancreas noninvasively. ${ }^{6}$

\section{Imaging Modalities}

\section{Radiographs}

Plain radiographs are rarely used these days to diagnose $\mathrm{CP}$. Usually done as a part of abdominal pain evaluation in these patients to rule out other causes such as bowel obstruction and perforation. If performed in these patients, it may show calcification that is noticed along the course of pancreas. The changes on radiographs are apparent only in the late stage of $\mathrm{CP}$.
License terms

$($ () (1) $\Theta \circledast$ 


\section{Ultrasonography}

Pancreas, being a retroperitoneal organ, the overlying gasfilled bowel loops and obese habitus posed a problem in its visualization. The modality is also operator dependent, however, using some maneuvers like asking the patient to drink water, so as to make the stomach distended providing a window, scanning the patient in oblique position and using spleen as the window to visualize the tail of the pancreas, asking the patient to bloat the abdomen, and scanning in sitting position can allow the visualization of whole of the pancreas. In initial stages of $\mathrm{CP}$, the gland may appear normal on USG; however, later it may show areas of either hypo- or hyperechogenicity representing inflammation or fibrosis in parenchyma, respectively. As the disease advances, there can be progressive atrophy of the gland with resultant irregular dilatation of main pancreatic duct (MPD) and side branches. USG may visualize calcifications with posterior acoustic shadowing within the pancreatic ducts or parenchyma. Various complications of $\mathrm{CP}$ like pseudocyst, thrombosis of splenoportal axis, and arterial pseudoaneurysms can be appreciated on USG. ${ }^{2}$ Elastography is relatively a new technique which can be used to qualitatively and quantitatively measure the stiffness of the pancreas which can have prognostic implications for surgery in these patients. ${ }^{7}$ Fibrosis, which is a part of $\mathrm{CP}$ evolution, increases the stiffness which can be measured using elastography. Also tumors developing in the background of CP can show increased stiffness which can be detected on elastography.

\section{Computed Tomography}

Noncontrast computed tomography (NCCT) is the modality of choice for detection of pancreatic calcification with a sensitivity of 60 to $95 \%$ in late stages of $\mathrm{CP}^{2}$ Alcoholism is the most common cause of chronic calcific pancreatitis which typically shows multifocal small calcifications with the degree of calcification usually proportional to duration of disease ( - Fig. 1).

Hereditary pancreatitis, tropical pancreatitis, and genetic mutation related $\mathrm{CP}$ has larger calcifications measuring more than 2 to $5 \mathrm{~cm}$, with bull's eye appearance described in hereditary pancreatitis. ${ }^{8}$ In patients with cystic fibrosis with cystic fibrosis transmembrane conductance regulator (CFTR) mutations, the calcifications appear late and if they occur, they are usually small compared with other genetic causes of $\mathrm{CP}^{8}$ (-Fig. 2).

Any obstruction to MPD can also lead to chronic pancreatitis; however, calcifications are rarely seen. The causes of obstructive CP can be periampullary carcinoma or stricture at ampulla of various causes. On imaging, pancreatic head is defined as the part of parenchyma within the duodenal C-practical landmark for cross-sectional the superior mesenteric vein (SMV; including the neck and the uncinate process). The pancreatic body extends from the left border of SMV till the left border of aorta and pancreatic tail from left border of aorta to the splenic hilum. The normal thickness of pancreas varies with age. The left margin of vertebral body is considered a practical landmark for cross-sectional imaging and superior mesenteric artery (SMA) is taken as landmark for USG. The normal measurements of head, body, and tail are 21,16 , and $7 \mathrm{~mm}$, respectively.

Multiphase CT protocol for CP include unenhanced scan, pancreatic phase (35-45 seconds after the start of contrast injection) and portal venous phase (55-70 seconds after the start of contrast injection). ${ }^{8}$ Pancreatic phase images show
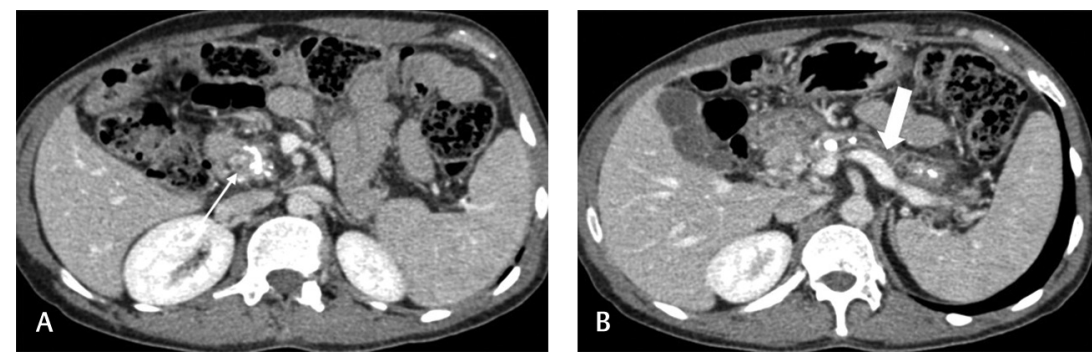

Fig. 1 (A, B) CECT of a patient who had a history of recurrent abdominal pain showing diffuse atrophy of pancreas. The MPD is dilated (thick arrow) and has multiple intraductal and parenchymal calcifications (thin arrow). These findings are diagnostic of chronic calcific pancreatitis. CECT, contract-enhanced computed tomography.
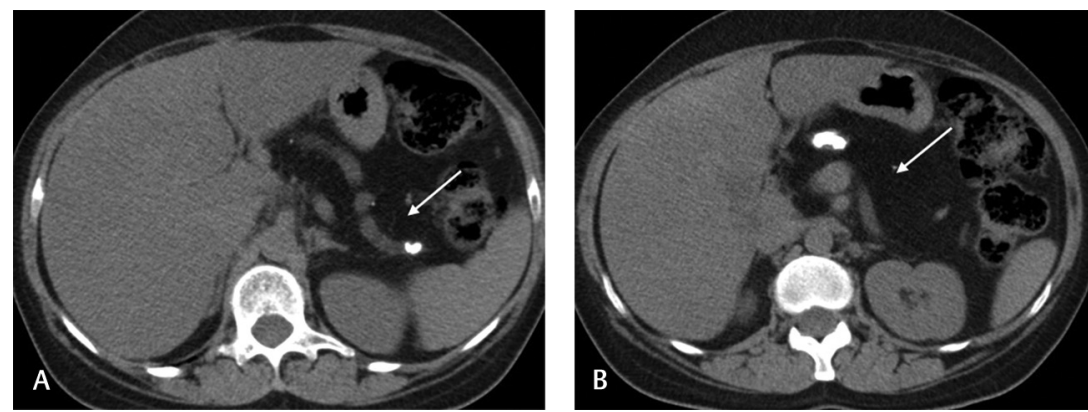

Fig. 2 (A, B) Noncontrast computed tomography (NCCT) abdomen of a patient diagnosed with cystic fibrosis showing atrophy and fatty replacement of the entire pancreas (arrow). The MPD is dilated with multiple calculi within. Fatty replacement of pancreas is because of chronic low grade inflammation which happens in cystic fibrosis. 
peak pancreatic parenchymal enhancement and therefore provide the best lesion to pancreas contrast. Portal phase images are helpful to assess the extent of venous involvement and to identify liver metastasis. Normal pancreas is homogeneous with lobulated outline. The characteristic features of CP are atrophy, ductal dilatation and calcification. Ductal dilatation is usually irregular and beaded in $\mathrm{CP}$ in contrast to smooth linear dilatation in pancreatic carcinoma.

$\mathrm{CT}$ also has the ability to detect the complications related to CP. Pancreatic pseudocysts are seen in 20 to $40 \%$ of cases in $\mathrm{CP}$ and the commonest cause is alcoholic pancreatitis ${ }^{9}$ (-Fig. 3).

Pseudocysts are cystic lesions with a well-defined wall and are commonly seen in peripancreatic, intrapancreatic, retropancreatic region or in remote locations such as chest, spleen, liver. Rupture of these pseudocysts can lead to fistula formation with peritoneal or pleural cavity. Pseudocysts and peripancreatic inflammation can cause phlebitis and thrombosis which are seen as thrombus in splenic vein, portal vein, SMA, and splenoportal axis leading to opening up of multiple collaterals. Inflammation in the arteries leads to formation of pseudoaneurysms. In CP gastroduodenal, pancreaticoduodenal, and splenic arteries are most commonly involved. Peripancreatic inflammation can also lead to formation of strictures in the common bile duct (CBD) with resultant intrahepatic biliary radicle dilatation (IHBRD).

\section{Magnetic Resonance Imaging}

MRI is a noninvasive imaging modality for the evaluation of pancreatic pathology and can accurately characterize various pancreatic lesions. For diagnostic purpose, MRCP has replaced ERCP for biliary and pancreatic ducts. It is a specialized MR technique in which heavily T2-weighted sequences are used to image fluid-filled structures without a need for contrast agent. MR has superior contrast resolution and does not use ionizing radiation. Pancreas is diffusely hyperintense on T1-weighted sequence due to the presence of aqueous proteins, abundance of endoplasmic reticulum in protein producing acinar cells, and the presence of paramagnetic ions such as manganese. ${ }^{10}$ It is one of the most T1 bright organs in the abdomen which can be better appreciated on fat saturated sequences. Hence pancreatic pathologies appear hypointense on T1-weighted sequence. Fibrosis in $\mathrm{CP}$ results in delayed enhancement in postcontrast fat saturated images, as well as restricted diffusion, as seen on apparent diffusion coefficient (ADC) maps of diffusion-weighted imaging (DWI). ${ }^{11} \mathrm{MRCP}$ has emerged as a noninvasive tool for ductal evaluation. The ductal changes are much better visualized on MRCP than on CT scan because of excellent contrast resolution. The ductal findings of early disease can range from normal looking MPD to mild irregularity of MPD and its side branches ( - Fig. 4).

There is progressive glandular atrophy and poor enhancement in portal venous phase and increased enhancement in delayed phase when the disease progresses. Severe ductal
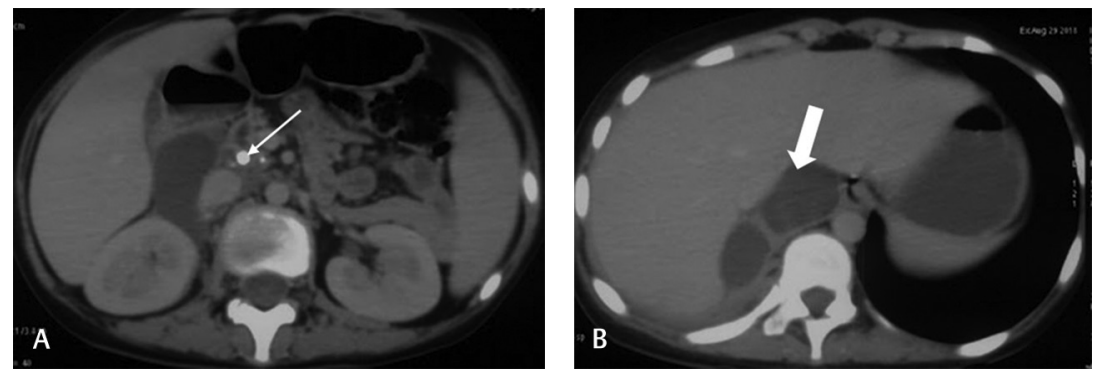

Fig. 3 (A, B) CECT of a follow-up patient of chronic calcific pancreatitis showing typical features such as atrophy and calcification (thin arrow). In addition, there is a fluid collection seen around the head of pancreas which is seen to extend cranially along the inferior vena cava reaching up to the subdiaphragmatic location (thick arrow). In the setting of chronic calcific pancreatitis, the fluid collection tracking across the tissue planes is suggestive of pancreatic pseudocyst. CECT, contract-enhanced computed tomography.
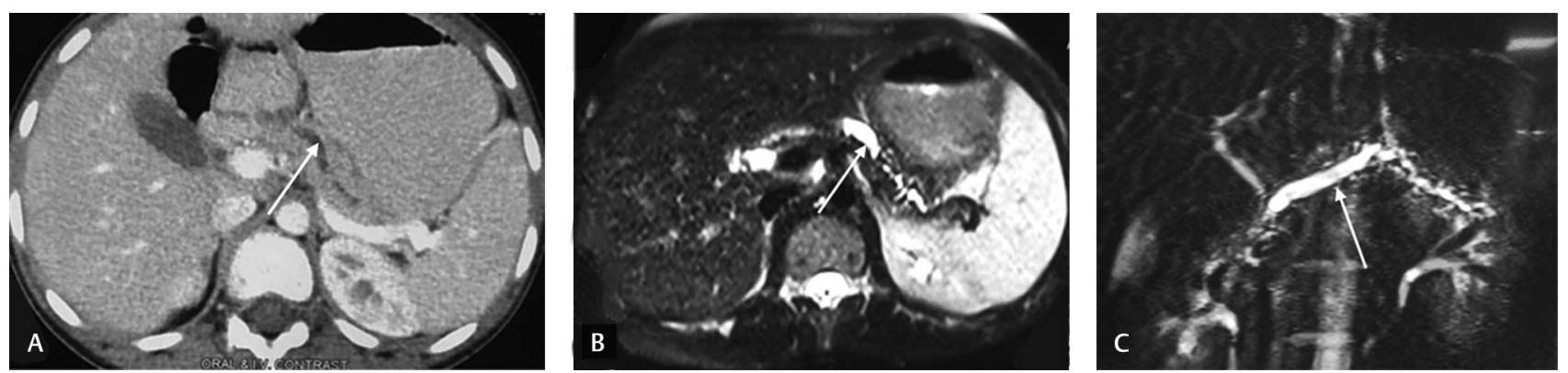

Fig. 4 A 10-year-old child presented with a long standing history of recurrent abdominal pain. CECT. (A) Mild atrophy of pancreas with dilated MPD (thin arrow). There was no calcification seen in the pancreas. T2-weighted fat saturated, (B) and thick section projectional MRCP, (C) images showing the dilatation of MPD (thin arrow) which is irregular and associated dilatation of side branches mainly in the body and tail region. This child was diagnosed with chronic pancreatitis. CECT, contract-enhanced computed tomography; MRCP, magnetic resonance cholangiopancreatography. 
changes in the form of irregular dilatation of both MPD and side branches along with interposed strictures gives the appearance of chain of lakes. Another advantage of MRCP is that it can detect various complications of $\mathrm{CP}$ such as pseudocyst and fistula and also in delineating congenital duct anomalies such as pancreatic divisum. ${ }^{8}$ One of the downsides of MRCP is that calcifications are not that well visualized as on NCCT.

Another situation where MRI scores over CT is the differentiation of carcinoma pancreas from mass forming chronic pancreatitis (MFCP) by demonstrating "duct penetrating sign" in the latter. ${ }^{12}$ This differentiation between these two entities is important because both of them present as pancreatic masses but the management is vastly different. S-MRCP has emerged as a tool to assess the exocrine function of the gland. ${ }^{13}$ Secretin is an enzyme secreted by " $\mathrm{S}$ " cells of the duodenum which facilitates the secretion of bicarbonate rich fluid from the pancreas. Patient should be fasting for 4 to 6 hours and a negative oral contrast is given 30 minutes before the study to suppress the signal of fluid in duodenum. Presecretin baseline images are obtained. Secretin is injected intravenously and series of T2-weighted images are acquired every 30 seconds for 15 minutes. Normal pancreatic duct shows maximum dilatation in 2 to 5 minute which is at least $1 \mathrm{~mm}$ greater than baseline. S-MRCP shows side branch ductal dilatation in CP due to fibrosis which otherwise may not be seen on conventional MRCP. The exocrine function is graded as follows: grade 1 is fluid confined to duodenal bulb, grade 2 is fluid seen in first and second part of the duodenum, while grade 3 is fluid seen till the third part of the duodenum. Grades 1 and 2 are considered by exocrine insufficiency.

\section{Endoscopic Ultrasonography}

Endoscopic USG (EUS) is an endoscopic modality which can overcome the issues associated with transabdominal USG such as poor window and resolution. High frequency, orally introduced transducer results in better visualization of the pancreas. The resolution is very high that it can detect early changes of $\mathrm{CP}$ even before the changes occur on ERCP which is considered as the gold standard for the diagnosis of $\mathrm{CP}^{4,14}$ Normal pancreas appears homogeneous on EUS and, in $\mathrm{CP}$, the gland can show heterogeneous appearance, tiny cystic changes, side branch ectasia, and increased echogenicity of main duct. It can be used along with USG-based contrast agents which can further improve the contrast resolution and help in the detection of tumors in the background of $\mathrm{CP}$. EUS-guided fine needle aspiration cytology (FNAC) and biopsy can also be performed which is an added advantage of this modality.

\section{Endoscopic Retrograde Cholangiopancreatography}

Once considered the gold standard for diagnosis of $\mathrm{CP}$ is now seldom used, instead MRCP is preferred for diagnosis of MPD and side branch dilatation, strictures, and filling defects in the ducts. Cambridge classification is used in ERCP for staging the $\mathrm{CP}$, while its modified version is used in MRCP. ${ }^{15}$ It depends on the status of MPD, number of side branches, presence of calculi, and ductal strictures.

\section{Unusual Types of Chronic Pancreatitis}

\section{Autoimmune Pancreatitis}

Autoimmune pancreatitis (AIP) is a rare form of chronic pancreatitis characterized by abundant lymphoplasmacytic infiltration, interstitial fibrosis, obliterative venulitis, and elevated serum immunoglobulin (Ig)-G4 levels. Patients present with chronic abdominal pain, weight loss, jaundice, and diabetes mellitus. Characteristic imaging features of AIP include sausage-shaped pancreas with a capsulelike rim and irregular narrowing of the MPD. Two subtypes of AIP are described which include: type I known as lymphoplasmacytic sclerosing pancreatitis (LPSP) which is characterized by elevated serum IgG4 levels, abundant infiltration of IgG4 positive cells on histopathology, and extrapancreatic involvement ${ }^{16}$ (-Fig. 5). Upper limit of normal serum IgG4 is $1.4 \mathrm{~g} / \mathrm{L}$ and two times rise is considered abnormal. Type 2 is also known as idiopathic duct centric pancreatitis (IDCP). ${ }^{16}$ Unlike LPSP, IDCP usually does not show rise in $\operatorname{IgG} 4$ and infiltration by $\operatorname{IgG} 4$ positive cells (-Fig. 6).

Biopsy can also be performed which can be associated with inflammatory bowel disease. On imaging, pancreas may show diffuse, focal, or multifocal involvement ( - Fig. 7).

Pancreas may show loss of normal lobular or feathery outline. A hypoattenuating rim on both T1- and T2-weighted sequences, which shows characteristic delayed enhancement is seen (-Fig. 5). There is also foreshortening of the tail of pancreas leading to rounded appearance of tail. The MPD shows diffuse or focal narrowing due to fibrosis in the gland and is referred to as ice pick sign. ${ }^{17}$ There can be mild enhancement in the wall of MPD as well. HISORt criteria, which has five cardinal features for the diagnosis of AIP, namely, histology, imaging, serological findings, other organ involvement, and response to steroid therapy, are used for the diagnosis of this entity..$^{18}$

The focal type of AIP can present as a mass like lesion in 33 to $41 \%$ of cases which needs to be differentiated from pancreatic carcinoma. Homogenous enhancement, duct penetrating sign, and low-ADC values help in differentiating mass forming AIP from adenocarcinoma. Combination of these criteria results in specificity of $98.7 \%$ which can help in avoiding unnecessary invasive procedures and major surgeries. Delayed enhancement in AIP due to fibrosis also helps in differentiating it from pancreatic adenocarcinoma. ${ }^{19}$ Also elevated carcinoembryonic antigen and cancer antigen 19 to 9 levels are raised in pancreatic adenocarcinoma but not in AIP.

\section{Groove Pancreatitis}

It is a rare type of focal chronic pancreatitis which affects the groove between the head of pancreas, duodenum, and CBD. It occurs predominantly in alcoholics. The term paraduodenal pancreatitis is the new term now used to represent groove pancreatitis, cystic dystrophy of duodenum, and paraduodenal wall cysts. Two types of paraduodenal pancreatitis are recognized-pure form and segmental form. In pure form only the groove is involved, 

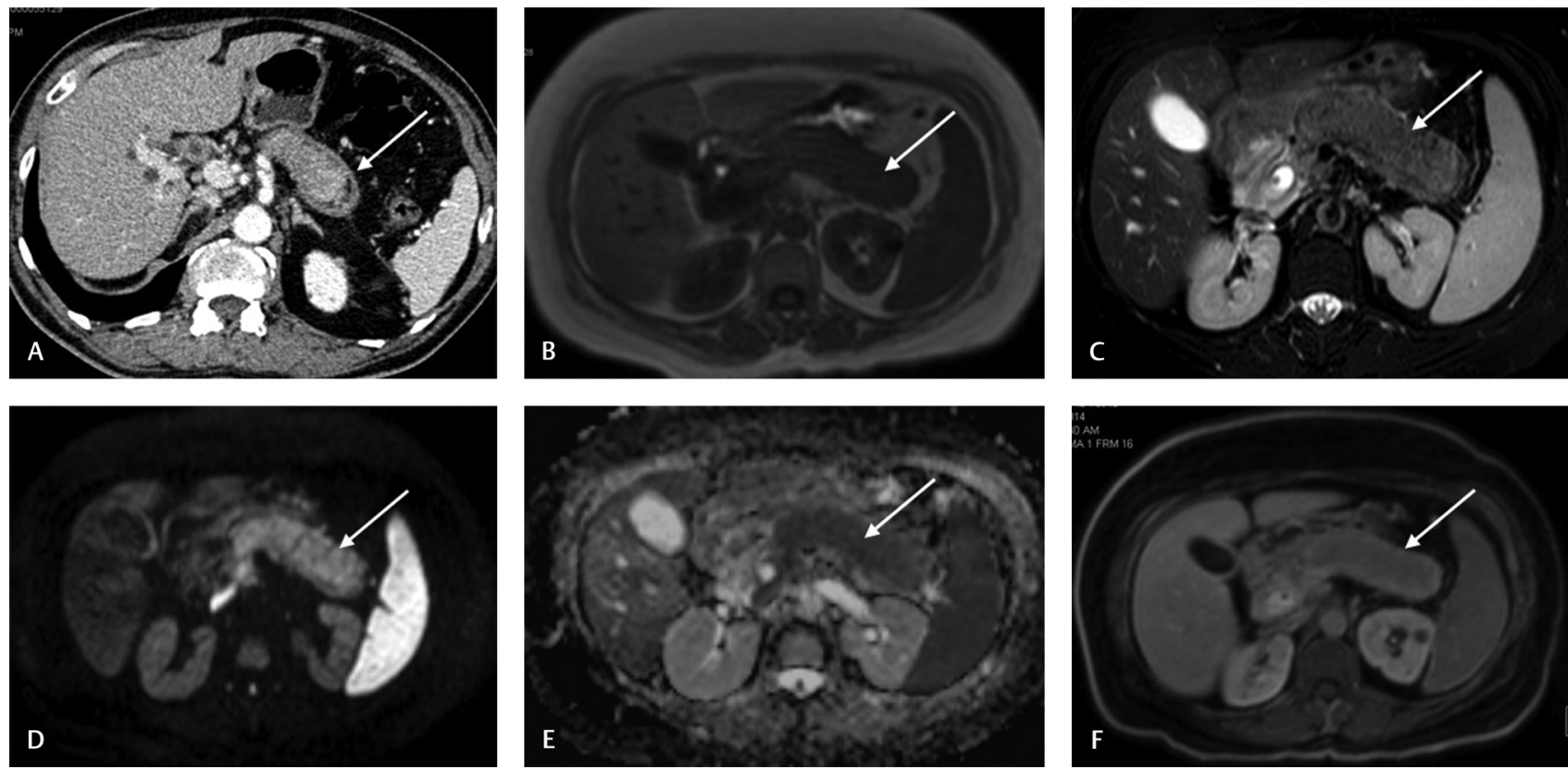

Fig. 5 CECT abdomen (A) of a female patient presented with chronic abdominal pain showing bulky pancreas (arrow) with a thin fibrotic rim around. There is associated mild intrahepatic biliary radicle dilatation as well. T1- and T2-weighted MRI image (B, C) showing loss of normal intensity of pancreas with bulky appearance (arrow). Diffusion weighted image (D) and apparent diffusion coefficient (ADC) map (E) showing diffusion restriction involving the entire pancreas. Postcontrast image $(\mathbf{F})$ in equilibrium phase showing enhancement of the fibrotic rim around the pancreas (arrow). It is important to note that MPD is not dilated. Patient also had elevated serum IgG4 level. The above features are typical for type- 1 autoimmune pancreatitis. CECT, contract-enhanced computed tomography; Ig, immunoglobulin; MRI, magnetic resonance imaging.
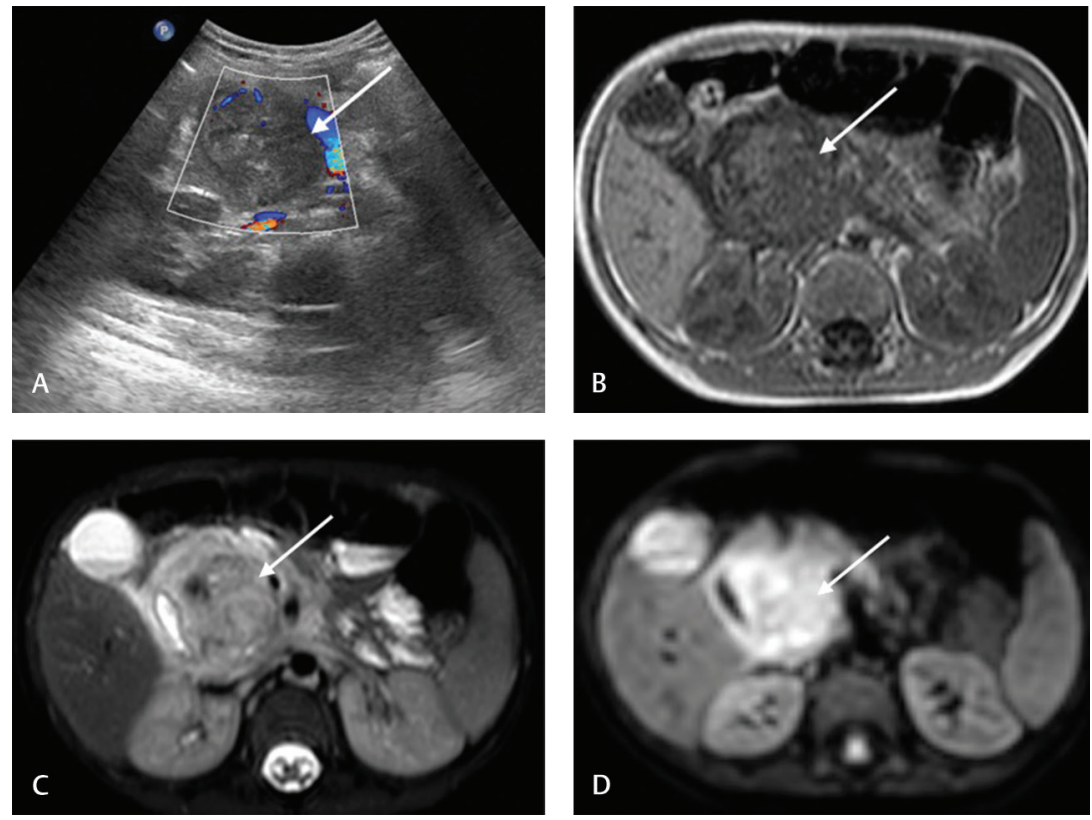

Fig. 6 USG (A) of a child who presented with abdominal pain showed a heterogeneous mass lesion (arrow) in the region of head of pancreas. (B) MRI was performed to evaluate further which showed a heterogeneous mass lesion arising from the head of pancreas (arrow) which was mildly hyperintense on T2-weighted image (C) causing mass effect over the duodenum and mesenteric vessels. The lesion showed significant diffusion restriction on DWI (D). The serum IgG4 level was not elevated and the lesion was sampled which showed type-2 autoimmune pancreatitis. DWI, diffusion-weighted imaging; Ig, immunoglobulin; MRI, magnetic resonance imaging; USG, ultrasonography.

while in segmental type, there is also involvement of head of pancreas along with the groove. ${ }^{20}$

On imaging sheet like soft tissue is seen in the groove which shows delayed enhancement due to fibrosis. Small cystic lesions along the medial wall of the duodenum can also be seen ( - Fig. 8 ).
The disease entity is a great mimicker of pancreatic adenocarcinoma. One way to differentiate these two conditions on imaging is that the groove pancreatitis displaces the gastroduodenal artery toward pancreatic head, while it is displaced toward the duodenum by pancreatic head carcinoma. $^{8}$ 

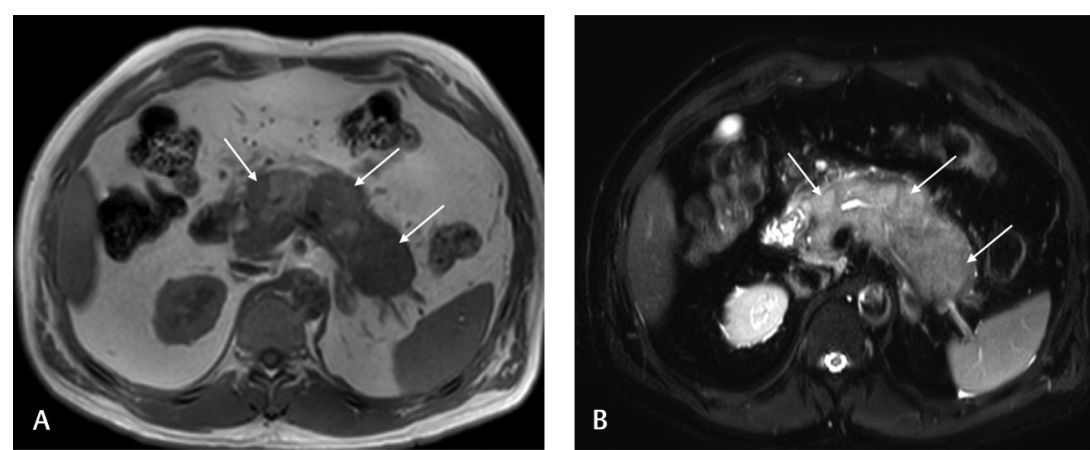

Fig. 7 (A, B) T1- and T2-weighted images of pancreas showing multiple focal lesions (arrows) causing lobulated outline and bulkiness of the pancreas. The intervening normal appearing parenchyma is visualized as a T1 hyperintense area in the neck region. This is also an ill-defined rim and fat stranding seen around the pancreas. Patient underwent EUS and one of the lesions was sampled. On the basis of imaging and histopathological features, diagnosis of type- 1 multifocal autoimmune pancreatitis was made. EUS, endoscopic ultrasound.
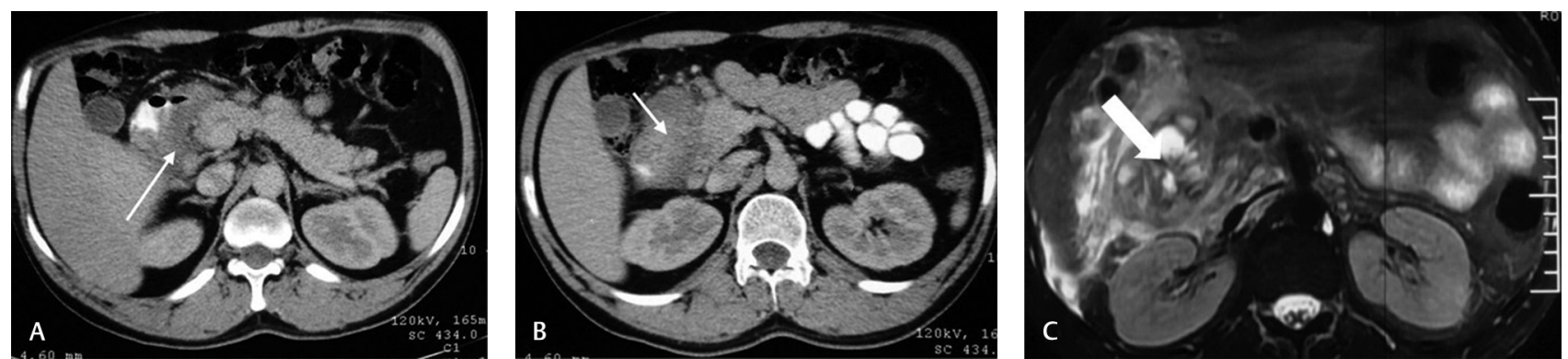

Fig. 8 (A-C) A middle-aged male patient who was an alcoholic presented with abdominal pain. CECT images (A, B) a plaque like mass (thin arrow) lesion between the duodenum and pancreatic head. No calcification or MPD dilatation was seen. T2-weighted MRI image (C) showed duodenal wall thickening with multiple cystic lesions in the wall (thick arrow) and associated inflammatory changes around. These changes are consistent with groove pancreatitis. MRI, magnetic resonance imaging.

\section{Mass Forming Chronic Pancreatitis}

MFCP is a rare chronic inflammatory disorder which can mimic pancreatic carcinoma, focal AIP, and groove pancreatitis both on clinical grounds and imaging. These lesions are often diagnosed on histopathology when these patients undergo Whipple's procedure. It is challenging to differentiate MFCP from carcinoma on contrast-enhanced CT (CECT), MRI, and even on positron emission tomography (PET) CT. Even EUS-guided sampling does not provide accurate diagnosis in all cases. However, there are few promising techniques such as $\mathrm{CT}$ perfusion has been used to differentiate MFCP from carcinoma pancreas..$^{21}$ Both MFCP and carcinoma pancreas showed decreased blood flow compared with normal pancreas on CT perfusion; however, the magnitude of reduction in blood flow was reported to be significantly lower on carcinoma pancreas compared with MFCP. In spite of these advancements in imaging techniques, EUS-guided sampling is still necessary in these patients.

\section{Chronic Pancreatitis and Pancreatic Carcinoma}

Pancreatic carcinoma can develop in 2 to $8 \%$ of patients suffering from CP. Although challenging, there are some imaging features which can help in differentiating these two entities. Change in the morphology of the pancreas like abnormal contour, alteration or disappearance of the pancreatic calcification, vascular invasion, and negative penetrating duct sign are some of the imaging markers to suspect pancreatic adenocarcinoma ( - Fig. 9).

In addition, newer imaging techniques such as perfusion imaging on CT and MRI, DWI on MRI, fluorodeoxyglucose (FDG)-PET CT, and shear wave elastography may help in their differentiation. However, at most of the times, either FNAC or biopsy is required for confirmation of diagnosis. 

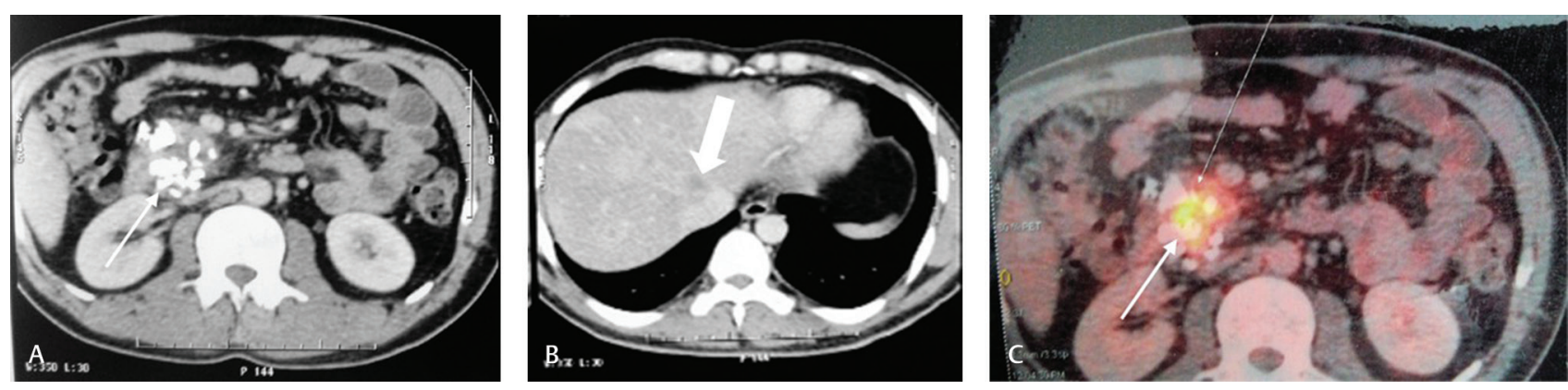

Fig. 9 (A-C) CECT images (A, B) of a patient who was on follow-up for CCP presented with weight loss and worsening of pain showed bulky appearance of head (thin arrow) with extensive calcifications and multiple focal lesions (thick arrow) in liver. The above features were suspicious for metastatic carcinoma head of pancreas developing in the background of CCP. FDG-PET (C) showed avid tracer uptake in the same area and EUS guided sampling of the lesion confirmed the diagnosis. CCP, chronic calcific pancreatitis; CECT, contract-enhanced computed tomography; EUS, endoscopic ultrasound; FDG-PET CT, fluorodeoxyglucose positron emission tomography.

\section{Conflict of Interest}

None declared.

\section{References}

1 Uc A, Andersen DK, Bellin MD, et al. Chronic pancreatitis in the 21st century - research challenges and opportunities: summary of a national institute of diabetes and digestive and kidney diseases workshop. Pancreas 2016;45(10):1365-1375

2 Kamat R, Gupta P, Rana S. Imaging in chronic pancreatitis: state of the art review. Indian J Radiol Imaging 2019;29(2):201-210

3 Lindkvist B. Diagnosis and treatment of pancreatic exocrine insufficiency. World J Gastroenterol 2013;19(42):7258-7266

4 Stevens T, Parsi MA. Update on endoscopic pancreatic function testing. World J Gastroenterol 2011;17(35):3957-3961

5 Hart PA, Topazian M, Raimondo M, et al. Endoscopic pancreas fluid collection: methods and relevance for clinical care and translational science. Am J Gastroenterol 2016;111(9):1258-1266

6 Matos C, Winant C, Delhaye M, Devière J. Functional MRCP in pancreatic and periampullary disease. Int J Gastrointest Cancer 2001;30(1,2):5-18

7 Sigrist RMS, Liau J, Kaffas AE, Chammas MC, Willmann JK. Ultrasound elastography: Review of techniques and clinical applications. Theranostics 2017;7(5):1303-1329

8 SharmaR, KandasamyD. Imaging in chronic pancreatitis. Pancreapedia Exocrine Pancreas Knowledge Base. Available at: https://www.pancreapedia.org/sites/default/files/DOI\%20 V3.\%20Imaging\%20in\%20chronic\%20pancreatitis\%20final\%20 revision\%20\%2024.6.15.pdf. Accessed February 17, 2020

9 Tan JH, Zhou L, Cao RC, Zhang GW. Identification of risk factors for pancreatic pseudocysts formation, intervention and recurrence: a 15 -year retrospective analysis in a tertiary hospital in China. BMC Gastroenterol 2018;18(1):143

10 Manikkavasakar S, AlObaidy M, Busireddy KK, et al. Magnetic resonance imaging of pancreatitis: an update. World J Gastroenterol 2014;20(40):14760-14777
11 Kamper L, Haage P, Brandt AS, et al. Diffusion-weighted MRI in the follow-up of chronic periaortitis. $\mathrm{Br} \mathrm{J}$ Radiol 2015;88(1052):20150145

12 Ichikawa T, Sou H, Araki T, et al. Duct-penetrating sign at MRCP: usefulness for differentiating inflammatory pancreatic mass from pancreatic carcinomas. Radiology 2001;221(1):107-116

13 Boraschi P, Donati F, Cervelli R, Pacciardi F. Secretin-stimulated MR cholangiopancreatography: spectrum of findings in pancreatic diseases. Insights Imaging 2016;7(6):819-829

14 Stevens T, Parsi MA. Endoscopic ultrasound for the diagnosis of chronic pancreatitis. World J Gastroenterol 2010;16(23):2841-2850

15 Kolodziejczyk E, Jurkiewicz E, Pertkiewicz J, et al. MRCP versus ERCP in the evaluation of chronic pancreatitis in children: Which is the better choice? Pancreas 2016;45(8):1115-1119

16 Kenji Notohara, Lizhi Zhang. Histology of autoimmune pancreatitis. Pancreapedia Exocrine Pancreas Knowledge Base. Available at: https://www.pancreapedia.org/sites/default/files/ logo_chapter_3_0.pdf. Accessed February 17, 2020

17 Martínez-de-Alegría A, Baleato-González S, García-Figueiras $\mathrm{R}$, et al. IgG4-related disease from head to toe. Radiographics 2015;35(7):2007-2025

18 Chari ST. Diagnosis of autoimmune pancreatitis using its five cardinal features: introducing the Mayo Clinic's HISORt criteria. J Gastroenterol 2007;42(Suppl 18) :39-41

19 Vijayakumar A, Vijayakumar A. Imaging of focal autoimmune pancreatitis and differentiating it from pancreatic cancer. ISRN Radiol 2013;2013(Jan)

20 Addeo G, Beccani D, Cozzi D, et al. Groove pancreatitis: a challenging imaging diagnosis. Gland Surg 2019;8(Suppl 3): S178-S187

21 Yadav AK, Sharma R, Kandasamy D, et al. Perfusion CT Can it resolve the pancreatic carcinoma versus mass forming chronic pancreatitis conundrum? Pancreatology 2016;16(6):979-987 